\title{
Physiological and Evolutionary Implications of Tetrameric Photosystem I in Cyanobacteria
}

\author{
Meng Li ${ }^{1,2, \dagger}$, Alexandra Calteau ${ }^{3}$, Dmitry A. Semchonok ${ }^{4}$, Thomas A. Witt ${ }^{1}$, \\ Jonathan T. Nguyen ${ }^{1}$, Nathalie Sassoon ${ }^{5}$, Egbert J. Boekema ${ }^{4}$, Julian Whitelegge ${ }^{6}$, \\ Muriel Gugger ${ }^{5}$, Barry D. Bruce ${ }^{1,2,7, *}$
}

\section{Affiliations:}

${ }^{1}$ Biochemistry \& Cellular and Molecular Biol. Dept., University of Tennessee, Knoxville, TN 37996, USA.

${ }^{2}$ Bredesen Center for Interdisciplinary Research and Graduate Education, University of Tennessee, Knoxville, TN 37996, USA.

${ }^{3}$ LABGeM, Génomique Métabolique, Genoscope, Institut François Jacob, CEA, CNRS, Univ Evry, Université Paris-Saclay, 91057 Evry, France.

${ }^{4}$ Electron Microscopy Dept., Groningen Biomolecular Sciences \& Biotechnology Institute, University of Groningen, 9700 AE Groningen, NLD.

${ }^{5}$ Collection of Cyanobacteria, Institut Pasteur, 75015 Paris, France.

${ }^{6}$ Pasarow Mass Spectrometry Laboratory, Geffen School of Medicine, University of California, Los Angeles, CA 90095, USA.

${ }^{7}$ Microbiology Dept., University of Tennessee, Knoxville, TN 37996, USA.

*Correspondence to: Barry D. Bruce (bbruce@utk.edu, +1-865-974-3380)

$\uparrow$ Current address: Institute of Biological Chemistry, Washington State University, Pullman, WA 99164, USA. 

5

\section{Abstract (146 words):}

Photosystem I (PSI) were reported as trimeric complexes in most characterized cyanobacteria, yet monomers in plants and algae PSI. Recent reports on tetrameric PSI raised questions regarding its structural basis, physiological role, phylogenetic distribution and evolutionary significance. In this study, by examining PSI in 61 cyanobacteria, we show that tetrameric PSI, correlating with a unique $p s a L$ gene and genomic structure, is widespread in the heterocyst-forming cyanobacteria and their close relatives. Physiological studies on these cyanobacteria revealed that tetrameric PSI is favored under high light, with an increased content of novel PSI-bound carotenoids (myxoxanthophyll, canthaxanthan and echinenone). Together this work suggests that tetrameric PSI is an adaptation to high light, along with results showing that change in PsaL leads to trimeric PSI monomerization, supporting the hypothesis of tetrameric PSI being the evolutionary intermediate in the transition from cyanobacterial trimeric PSI to monomeric PSI in plants and algae.

Key words: Cyanobacteria, Evolution, High Light Adaptation, Photosystem I, Tetrameric PSI 


\section{Introduction}

17 Photosystem I (PSI) is integral in the light reactions of oxygenic photosynthesis in cyanobacteria, 18 algae and plants (Golbeck, 2007). Upon illumination, PSI accepts an electron from plastocyanin or cytochrome $\mathrm{c}$ and transfers this electron to its major acceptor, ferredoxin. While algal and plant PSI have been reported to exist as mostly monomers (Ben-Shem et al., 2003; Kouril et al., 2005; Gardian et al., 2007; Veith and Buchel, 2007; Watanabe et al., 2011) and recently as dimers in spinach thylakoid membrane (Wood et al., 2018), cyanobacterial PSI has been reported as trimeric in most studies. The first study that showed this trimeric PSI structure was done in the cyanobacteria Synechococcus (Boekema et al., 1987). Later, trimeric PSI has been observed in a diverse range of filamentous and unicellular cyanobacteria (Shubin et al., 1992; Shubin et al., 1993; Tsiotis et al., 1995; Garczarek et al., 1998; Tucker and Sherman, 2000; Boekema et al., 2001; Bibby et al., 2003; Casella et al., 2017; MacGregor-Chatwin et al., 2017), including the most primitive known cyanobacterium, Gloeobacter violaceus PCC 7421 (Mangels et al., 2002). Early protein crystallography structures corroborated these findings of trimeric PSI in cyanobacteria (Almog et al., 1991; Jordan et al., 2001), such as the structure of trimeric PSI from the thermophilic cyanobacteria Thermosynechococcus elongatus BP-1 (Jordan et al., 2001). Due to these early seminal reports, it was assumed that PSI in all cyanobacteria was in a trimeric configuration, as opposed to the monomeric form found in plants and algae. Recently, a tetrameric form of PSI observed in the cyanobacteria Nostoc sp. PCC 7120 (Watanabe et al., 2011; Watanabe et al., 2014) and Chroococcidiopsis sp. TS-821 (TS-821) (Li et al., 2014; Semchonok et al., 2016; Shelaev et al., 2018) challenged this preconceived notion of PSI trimer being the sole oligomer in cyanobacteria. Yet, the PSI tetramer has not been viewed as a major oligomeric state in cyanobacteria. Although the discovery of tetrameric PSI in two species is now accepted, the mechanism controlling the oligomeric assembly and stability is not known. Furthermore, the physiological and evolutionary significance of this structural change has yet to be elucidated. A recent cryo-EM structure of PSI tetramer from TS-821 showed that tetrameric PSI is actually a dimer of dimers with two different interaction interfaces between monomers (Semchonok et al., 2016). This structure suggests subtle changes in the placement of the central subunit PsaL, yielding changes in helical bundling that has been implicated to be critical in the formation of PSI trimers. 
46 In light of the recent discovery of tetrameric PSI, we extended the study to encompass a much

47 larger and more diverse set of cyanobacteria to characterize the oligomeric state of PSI structure

48 throughout the cyanobacterial phylum. To investigate an underlying mechanism for tetrameric

49 PSI formation, we probe the correlation between PSI oligomeric states, PsaL sequence and

50 genomic structure using bioinformatics and biochemistry techniques. Finally, although the

51 physiological significance of the PSI tetramer was poorly understood in cyanobacteria, we have

52 explored several growth conditions that may alter the formation of the tetramer. Our findings

53 indicate that exposure to high light induces a significant increase in PSI tetramer formation,

54 while also increasing carotenoid content. These results shed light on the role and function of

55 higher order oligomers of PSI and provide insight into the possible cyanobacterial lineage

56 associated with the origin of the plastid. 


\section{Results}

\section{PSI Oligomeric States in Cyanobacteria}

To understand how PSI evolved from its cyanobacterial multimeric forms to the monomeric form in algae and plants, a comprehensive and unbiased understanding of the cyanobacterial PSI oligomeric forms is needed. This needs the investigation of the PSI structures through the diversity of cyanobacteria. Here, we have investigated the oligomeric states of PSI in 61 cyanobacteria including 34 heterocyst-forming cyanobacteria and five unicellular close relatives, as well as a set of 22 widely divergent unicellular and filamentous cyanobacteria for comparison (Dataset S1). For most heterocyst-forming cyanobacteria and their close unicellular relatives (HCR), tetrameric and/or dimeric PSI, besides the abundant monomeric PSI, was observed as the major PSI oligomers determined by BN-PAGE analysis (Figs 1, S2). Similarly, analysis of $T$. elongatus BP-1 and Synechocystis PCC 6803 consistently observed trimeric PSI by BN-PAGE (Figs 1a, S2). Specifically, 30 out of 34 heterocyst-forming cyanobacteria including poorly studied species such as Spirirestis rafaelensis UTEX B 2660 (Figs 1a, S2n), appeared to have tetrameric or dimeric PSI (Dataset S1). To verify that the altered electrophoretic mobility was truly indicative of the PSI tetramer, PSI oligomers for several species were examined using TEM, which confirmed their tetrameric structure (Figs 1b, S2). Surprisingly, a few heterocystforming cyanobacteria, Anabaena inaequalis UTEX B381, Calothrix membranacea UTEX B379, and Cylindrospermum licheniforme UTEX B2014 possessed only monomeric PSI (Fig. S2u). However, Nostoc sp. PCC 9335 represents the only species of cyanobacteria observed that had significant amounts of both trimeric and dimeric forms of PSI (Fig. S3a). Analysis of the unicellular cyanobacteria that are closely related to the heterocyst-forming cyanobacteria (Shih et al., 2013) including TS-821, Synechocystis sp. PCC 7509 and Gloeocapsa sp. PCC 7428 (Fig. S2a, b), exhibit more tetrameric, dimeric, and monomeric PSI than trimeric PSI. The two other species of Chroococcidiopsis that we tested, Chroococcidiopsis thermalis PCC 7203 (Fig. 1a, c) and Chroococcidiopsis sp. PCC 7434 (Fig. S3a), contained primarily monomeric PSI with some dimeric PSI. Collectively, these results revealed that besides monomeric PSI, tetrameric and dimeric PSI, instead of trimeric PSI, comprise the majority of PSI oligomers in the HCR. Trimeric PSI was either not detectable or only present as a minor species in this clade (Dataset $\mathrm{S} 1$ ), suggesting that there is a structural and potentially physiological basis for the propensity of dimeric/tetrameric PSI for these related cyanobacteria. 


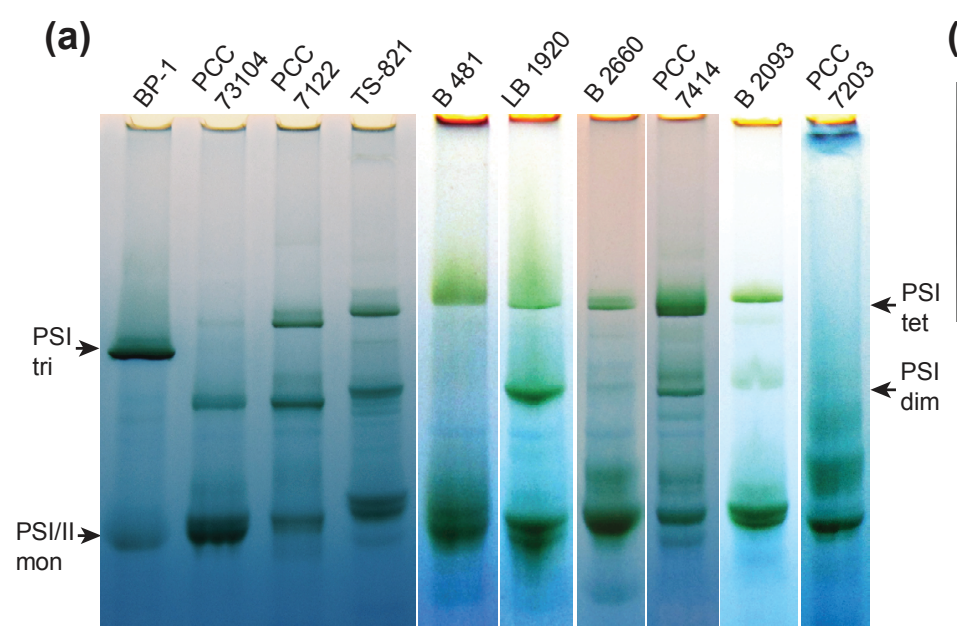

(c) subunits are colored and labeled. Scale bars: $10 \mathrm{~nm}$. (b)
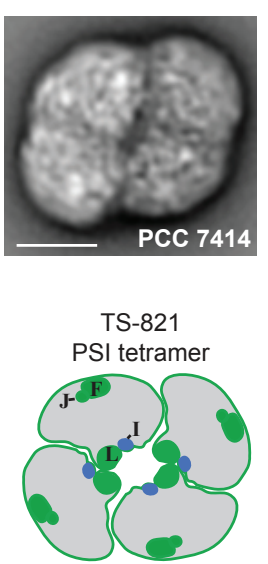

(d)

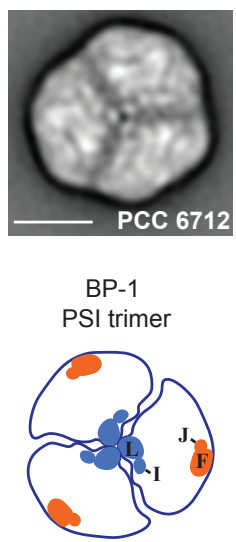

Fig. 1. Examples of BN-PAGE and TEM analyses of PSI oligomeric states in different cyanobacteria. (a) BN-PAGE analyses of the PSI oligomeric states of various heterocystforming cyanobacteria and their close relatives. (b) TEM structure of PSI tetramer from PCC 7414 compared with TS-821 PSI tetramer model. (c) BN-PAGE analyses of the PSI oligomeric states of cyanobacteria that are distantly related to heterocyst-forming cyanobacteria. (d). TEM structure of PSI trimer from PCC 6712 compared with BP-1 PSI trimer model. In $\mathbf{a}$ and $\mathbf{c}$, for each strain, the solubilization result of $0.4 \mathrm{mg} / \mathrm{mL} \mathrm{Chl}$ in $1 \% \mathrm{n}$-dodecyl- $\beta$-maltoside (DDM) is shown. TS-821, Synechocystis sp. PCC 6803 (PCC 6803), and T. elongatus (BP-1) are used as controls. The approximate migration distances for photosystem monomer (mon), dimer (dim), trimer (tri), tetramer (tet), and hexamer (hex) are indicated by arrows. The uncertainty was marked by '?' symbol. In b and d, the locations of PsaL (L), PsaI (I), PsaF (F), and PsaJ (J) 
104 In contrast to HCR, most other cyanobacteria that we studied contained predominantly trimeric

105

106

107

108

109

110

111

112

113

114

115

116

117

118

119

120

121

122

123

124

125

126

127

128

129

130

131

132

133

PSI (Figs. 1c, S2, S3; Dataset S1). When we investigated this PSI oligomer by TEM, we observed that this form was a clear trimer as shown for PCC 6712 (Fig. 1d). Amongst this group of PSI trimer-containing cyanobacteria, T. elongatus and Synechocystis PCC 6803 were included, within which the existence of trimeric PSI has been confirmed via crystallography (Jordan et al., 2001; Malavath et al., 2018). Many of the other cyanobacteria studied were also observed to contain trimeric PSI (Figs 1c, S2, S3; Dataset S1).

Occasionally, irregular PSI oligomers were observed in these diverse cyanobacteria based on results obtained via BN-PAGE analysis. For example, a potential PSI hexamer was observed in Chroococcidiopsis sp. PCC 6712 (Figs 1c, S2c, d) in equilibrium with the primary PSI trimer in vitro (Figs 1d, S2c, d). Although the BN-PAGE reveals some other minor PSI oligomeric forms in these strains, such as the PSI dimer in Microcystis sp. PCC 7806 (Figs 1c, S3a, b; Dataset S1), and a potential PSI tetramer in Planktothrix paucivesiculata PCC 8926 (Fig. S3c), the predominant PSI oligomer in these diverse cyanobacteria is trimeric. In contrast to other PSI trimer-containing strains, the PSI monomer was clearly dominant in Chamaesiphon minutus PCC 6605, along with Leptolyngbya sp. PCC 7124, showing significant amount of PSI dimers and monomers (Figs 1c, S3a).

\section{Correlation of PsaL and PSI Oligomers}

Previous structural studies on cyanobacterial PSI identified different PsaL-PsaL interactions within the trimeric and tetrameric PSI oligomers, shown schematically in Fig. 1b, d (Jordan et al., 2001; Semchonok et al., 2016). Previous work has identified not only subtle changes in the protein sequence of the PsaL subunits, but also a change in the genomic organization pertaining to where $p s a L$ is found in the cyanobacterial genome for TS-821 ( $\mathrm{Li}$ et al., 2014). Extending this observation to the larger group of cyanobacteria presented here, we are now able to see if this correlation holds true across the different PSI oligomeric forms identified. To allow visualization of the distribution of PSI oligomers within cyanobacteria, we localized the distribution of $p s a L$ in relation to psaI, psaF, and psaJ genes on a species tree (Figs 2, S4a). We analyzed the psaL distribution of cyanobacteria with a known genomic dataset and 14 additional heterocyst- 


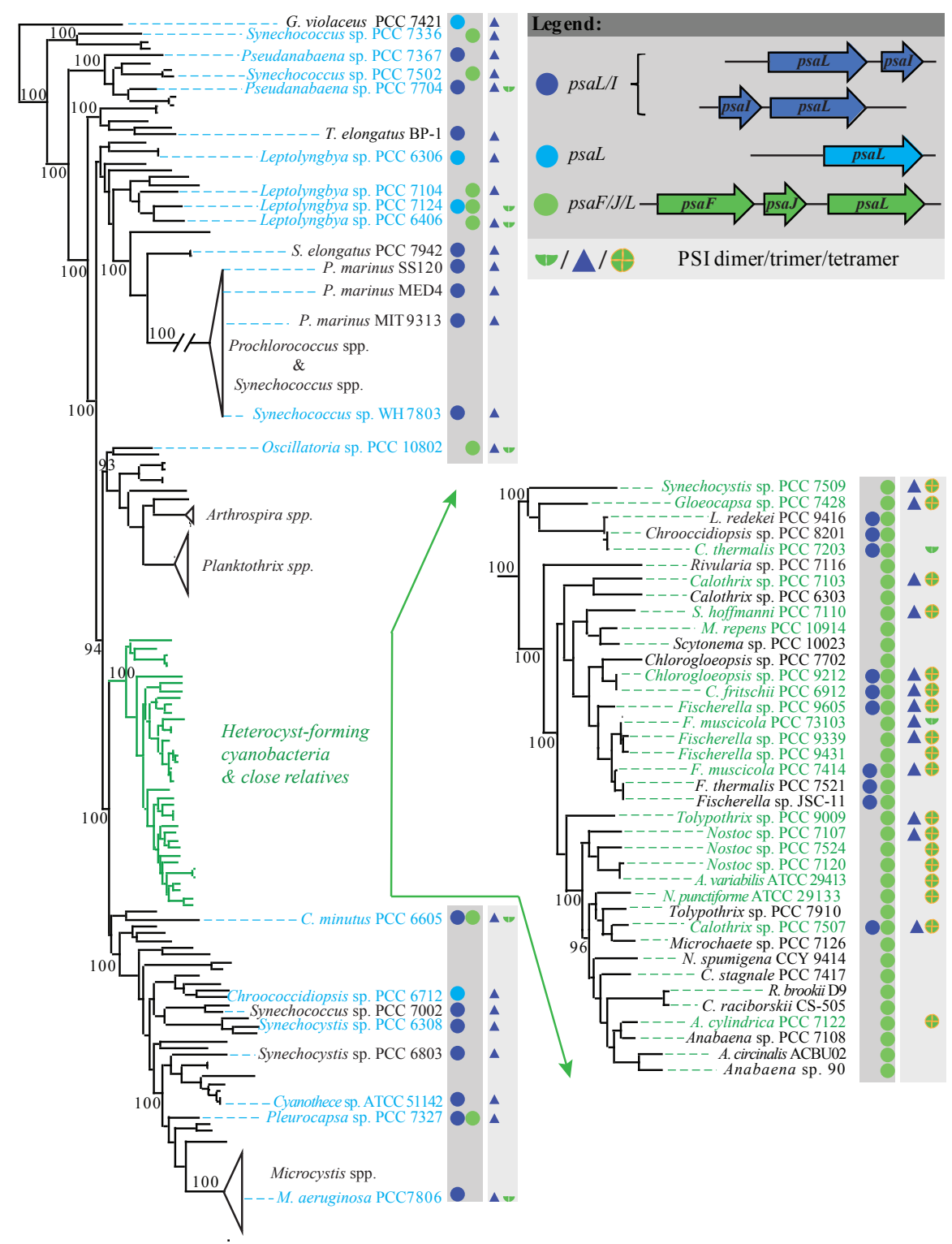

135 Fig. 2. Distribution of PSI oligomers and the location of psaL genes among cyanobacteria

136 with sequenced genomes. The relative location of psaL to psaI, psaF and psaJ genes are color

137 coded after each strain number, followed by the observed or reported oligomeric states of PSI in

138 that cyanobacterium. The clade including heterocyst-forming cyanobacteria and their close

139 relatives is expanded for clarity. Bootstrap values greater than or equal to $70 \%$ are indicated by

140 black dots. Strains whose PSI oligomeric states investigated in this study are colored in cyan or

141 green. A fully expanded species tree is shown in Fig. S4.

142 
143 forming strains. In all HCR, we observed the $p s a L$ gene located downstream of $p s a F$ and $p s a J$

$144(\mathrm{psaF} / \mathrm{J} / \mathrm{L})($ Figs 2, S4b), in agreement with an earlier report (Li et al., 2014). This PsaL encoded

145 by $p s a F / J / L$, along with prevailing tetrameric/dimeric PSI, clearly delineates the clade of

146 cyanobacteria, namely HCR, represented by green lines and the inset of Fig. 2.

147 Cyanobacteria that fall outside of the HCR clade often have the $p s a L$ flanked by $p s a I$ ( $p s a L / I)$ or 148 in a few cases found in $p s a F / J / L$ or isolated from other $p s a$ genes all together (Figs 2, S4a).

149 Besides the 9 strains with reported PSI trimers, our analyses of the PSI oligomers identified an 150 additional 16 strains with trimeric PSI using BN-PAGE (shown in blue text, Fig. 2). These PSI trimer-bearing cyanobacteria, spreading out in the cyanobacteria phylum outside HCR, cover each major branching point and clade. This group also includes the well characterized reference strains of T. elongatus and Synechocystis PCC 6803. When the absence of PsaL, coded by $p s a F / J / L$, is clearly correlated to the PSI trimer in cyanobacteria (Fig. 2), the presence of such PsaL does not always point to a tetrameric/dimeric PSI. The phylogenetic analysis of PsaL revealed that PsaL coded by $p s a F / J / L$ fall in to different lineages, with the ones in HCR being a unique clade (dark green in Fig. S4b). 7414 , also have two copies of the $p s a L$ gene, one copy organized as a $p s a F / J / I$ and the second as a $p s a L / I$ (Figs 2, S4a). The first copy (as in $p s a F / J / I$ ) points to HCR and having tetrameric/dimeric PSI (Fig. S4b, colored in dark green) while the second form of PsaL (in the psaL/I locus) is closely related to the recently identified far-red light responsive PsaL, found in monomeric PSI, in Leptolyngbya sp. strain JSC-1 (Fig. S4b, colored in red) (Gan et al., 2014). This indicates that some of the HCR may have the second copy of the $p s a L$ gene (in $p s a L / I$ ) being expressed during far-red light acclimation. tetramer, subunit analyses for the isolated PSI trimer and tetramer from three different cyanobacteria, TS-821, PCC 7428, and PCC 7414, were performed using LC-MS/MS. Interestingly for all three species, it is the same PsaL protein, encoded by $p s a L$ in $p s a F / J / L$, that was observed in both the tetrameric and trimeric PSI of these three strains (Fig. S5). Moreover, genome sequencing of TS-821 revealed only a single form of psaL. Although PCC 7414 encodes two copies of the $p s a L$ gene (Fig 2a), only the PsaL encoded by $p s a L$ in the $p s a F / J / L$ structure 
reinforced the possibility that the psaL encoded in psaL/I in PCC 7414 is an example of far-red light responsive PSI genes (Gan et al., 2014; Gan and Bryant, 2015). These far-red light responsive genes are organized differently in the genome. Moreover, when we build a maximum-likelihood tree of PsaL, the group of far-red light responsive forms of PsaL form a distinct clade (in red text) as shown in Fig. S4b. Phylogenetic analysis revealed the PsaL within the $p s a F / J / L$ loci of the HCR forms a single clade. However, the observation that the same PsaL is observed in both tetrameric and trimeric PSI in TS-821, PCC 7428, and PCC 7414 suggests that the unique PsaL encoded in $p s a F / J / L$ is necessary, yet not sufficient, to direct PSI tetramer formation.

\section{PsaL Replacements affect PSI Oligomerization}

To investigate precisely what structural property of PsaL is key to the determination of the PSI oligomeric state, we performed gene replacement experiments in the mesophilic cyanobacteria Synechocystis sp. PCC 6803. Replacing WT PsaL in Synechocystis sp. PCC 6803 by homologous recombination with either the TS-821 PsaL or Arabidopsis PsaL resulted in monomeric PSI in both mutants (Fig. 3a, b). When western blotting was performed on these monomeric forms of PSI, it was clear that they too contained PsaL bound to PSI (Fig. 3c, d). This observation suggests that the monomerization of PSI in these transgenic lines is not due to the lack of PsaL expression and/or assembly. These findings suggest that subtle changes in the PsaL structure alone can lead to the monomerization of trimeric PSI in cyanobacteria, as found with the predominant monomeric PSI in PCC 6605 (Figs 1c, S3a).

Our earlier work on TS-821 speculated that the loop sequence of PsaL between the second and third transmembrane helices might be a key part in PSI oligomer and PsaL evolution (Li et al., 2014). While this loop sequence structure was not resolved in the trimeric PSI crystal structure from T. elongatus (PDB 1JB0), later improved plant PSI monomer structures showed the interaction between this loop sequence with PsaH (Qin et al., 2015, Mazor et al., 2015). Noticing

200 that TS-821 PsaL has an unusual multi-proline motif in this loop sequence, we examined the 201 conservation of this loop sequence between PsaL second and third transmembrane helices for all 
(a)

(c)

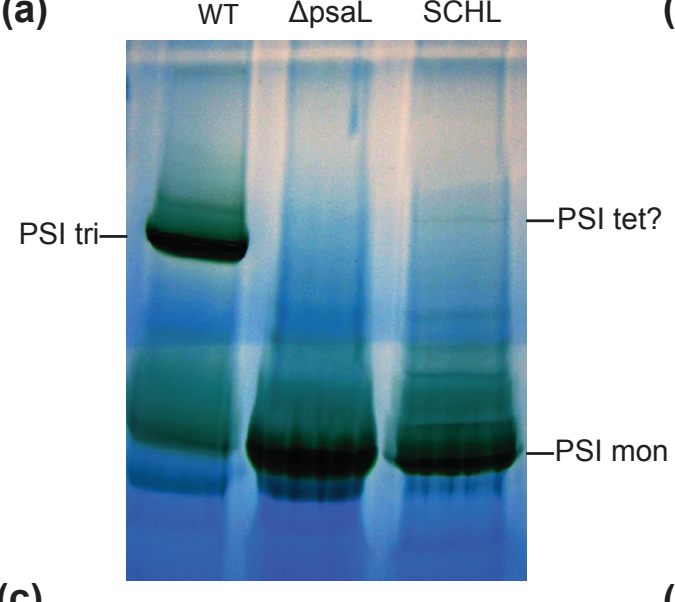

(d)
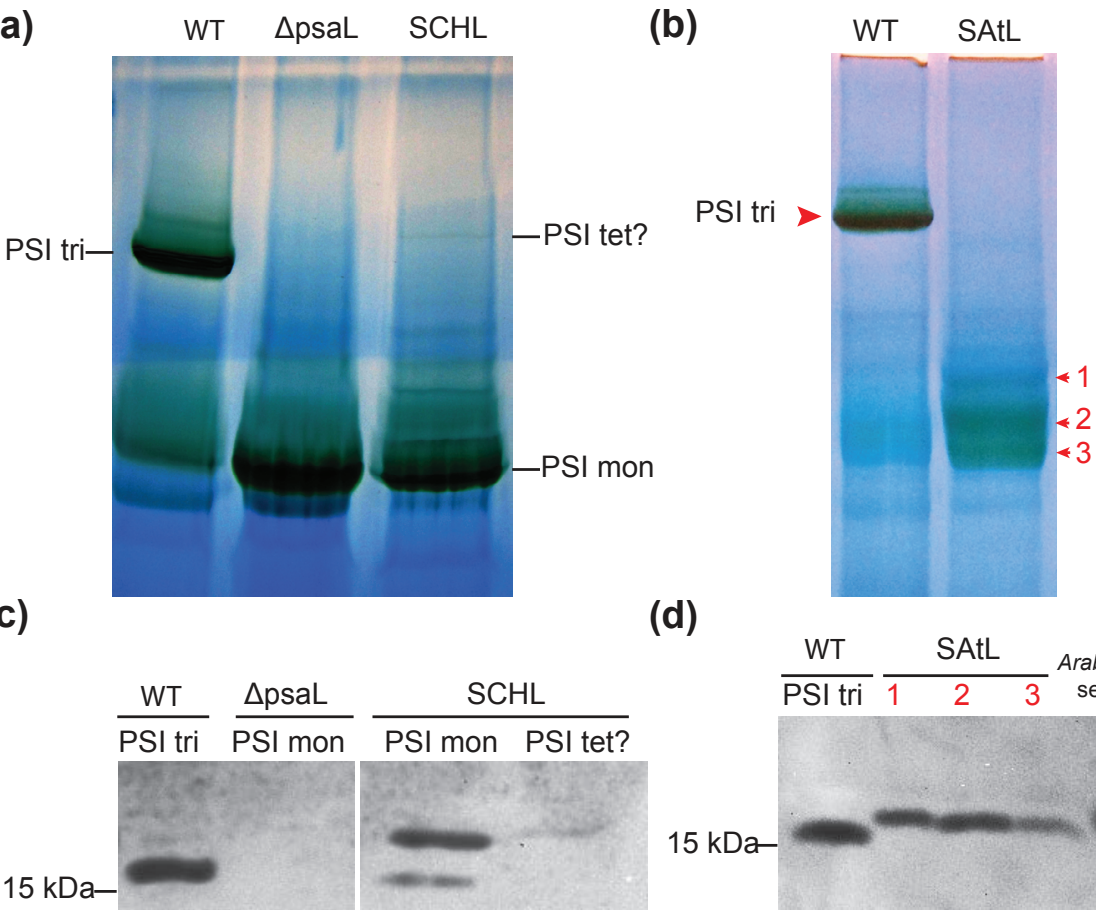

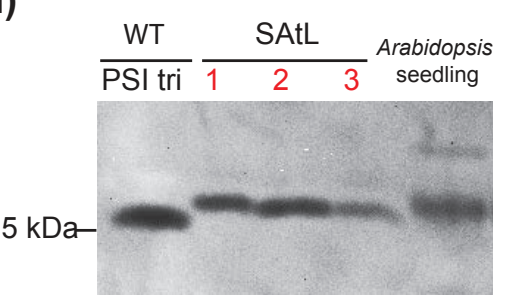

Fig. 3. BN-PAGE and western blot analyses of PSI oligomers in Synechocystis sp. PCC 6803 expressing different PsaL. (a) BN-PAGE of solubilized thylakoid membranes from Synechocystis sp. PCC 6803 WT, ApsaL, and expressing TS-821 PsaL (SCHL). PSI samples were sliced out for from the gel for following SDS-PAGE and western blot to detect the presence of PsaL. Each lane was loaded with solubilized membrane containing $16 \mu \mathrm{g}$ Chl. (b) BN-PAGE

210 of solubilized thylakoid membranes from Synechocystis sp. PCC 6803 WT and mutant expressing Arabidopsis thaliana PsaL (SAtL). (c)

(c) Western blot of the PSI samples from BN-

212 PAGE gel a. PSI trimer (tri) from WT, PSI monomers (mon) from $\triangle p s a L$ and SCHL, as well as 213 potential PSI tetramer (tet?) were analyzed. (d) Western blot of the PSI bands isolated from BN214 PAGE gel b. Arabidopsis thaliana seedling whole plant was used as a control. 
regions of PsaL from all studied 38 of the HCR, we identified a conserved proline-rich motif,

217 often as NPPxP followed by PNPP (Fig. S6). Many of the trimeric PSI containing sequences lack

218 this motif. However, interestingly this motif is shared by both copies of PsaL in PCC 6605 (Fig.

219 S6) which has predominantly monomeric PSI (Figs 1c, S3a). These observations suggest a

220 similar mechanism of trimeric PSI destabilization in PCC 6605 involving a similar multi-proline

221 motif as observed in this part of PsaL in the heterocyst-forming cyanobacteria.

\section{Influence of Environmental Factors}

224 It is now clear that the formation of PSI tetramers is restricted to a specific subset of cyanobacteria. Despite our ability to "map" this trait to the HCR, there was no clear understanding as to the physiological or evolutionary role that drives this change in oligomerization. Since most heterocyst-forming cyanobacteria have tetrameric PSI, the impact of nitrogen sources and presence of heterocysts on PSI tetramer formation was investigated. We grew three heterocyst-forming strains (PCCC 7414, PCC 7120, and PCC 7122) in both nitrate depleted BG-11 media (BG-110) and BG-11 supplemented with $\mathrm{NH}_{4}{ }^{+}$. Based on the similar PSI pattern observed with BN-PAGE (Fig. S7a) as observed in those cultured in standard BG-11 media (Fig. S2j, k), it indicates that the nitrogen sources did not affect the presence of tetrameric PSI. Similar result was also observed for Fischerella sp. UTEX LB 1829 (BG-11 0 vs BG-11, Fig. $\mathrm{S} 2 \mathrm{e})$. These strains did not develop heterocysts with supplemented $\mathrm{NH}_{4}{ }^{+}$, indicating the presence of tetrameric PSI in vegetative cells. The presence of tetrameric PSI in unicellular strains such as TS-821, PCC 7509 and PCC 7428 (Figs 1a, S2a, b) also agrees with a PSI tetramer independent from heterocyst development, even though tetrameric PSI was shown in heterocysts (Cardona et al., 2009). In the case of Nodularia PCC 73104, the small amount of PSI tetramer was only observed in BG-11 medium (Fig. S2j), while the addition of $\mathrm{NH}_{4}{ }^{+}$or salt in the culture media appeared to diminish the tetrameric PSI (Fig. S7c). The special case of Nodularia PCC 73104 needs more studies to elucidate the significance of such response, since Nodularia are usually found in brackish or oceanic environments with some strains do not abolish their heterocysts in the presence of $\mathrm{NH}_{4}{ }^{+}$(Vintila and El-Shehawy, 2007; Vintila and El-Shehawy, 2010). In addition to nitrogen source, growth temperature had little, if any, effect on the presence of tetrameric or trimeric PSI (Fig. S7b) on the four different cyanobacteria investigated. For the impact of salinity, two strains of Nodularia, heterocyst-forming cyanobacteria isolated from 
we observed little change in the amount of the tetramer/dimer (Fig. S7c). At the same time, salinity did not affect the presence and amount trimeric PSI in Pseudanabaena sp. B SP48 (Fig. $\mathrm{S} 3 \mathrm{e})$. Together these results suggest that none of the factors presented above, i.e, nitrogen source, heterocyst development, temperature stress, and salinity, has any effect on the formation of tetrameric and trimeric PSI.

\section{PSI Tetramer in Response to Light Intensity}

255 In contrast to other environmental factors, upon altering the light intensity during growth we saw a clear effect on the ratio of the PSI oligomeric forms. Our study on TS-821 showed that in response to light intensity change from low light (LL) to high light (HL), the tetrameric PSI accumulated, while the PSI trimer decreased (Semchonok et al., 2016). To confirm that light intensity was a common factor determining PSI tetramer/trimer ratio, we investigated the HL

260 response of additional cyanobacterial strains. We observed an increase in quantity and stability of the tetrameric PSI for both PCC 7428 and PCC 7414 under HL condition (Fig. 4). In addition, it was clear that the amount of trimeric PSI was much higher for PCC 7428 when grown at low light (LL) levels (Fig. 4a, b). The difference observed in PCC 7414 under HL versus LL was slightly less than that observed in PCC 7428 (Fig. 4c, d) possibly due to self-shading within the intertwined filaments in PCC 7414 (Fig. S8). Yet these results point to a common HL response, accumulating tetrameric PSI and suppressing trimeric PSI in strains having both forms of the PSI oligomers. Therefore, it is reasonable to speculate that tetrameric PSI is better adapted to HL than trimeric PSI.

We have performed a more detailed study of PSI oligomer profiles for TS-821 cultured under several different light intensities, which supported the hypothesis that tetrameric PSI is an adaption to HL. As shown in Fig. 5, the stability and relative quantity of the PSI tetramer in TS821 increased as the intensity of light is raised from $50-800 \mu \mathrm{mol} / \mathrm{m}^{2} / \mathrm{s}$. (Fig. 5a-g). When the relative quantities of PSI tetramer (percentage over all PSI oligomers) was plotted versus light intensities, it was clear that the apparent maximum increased with light intensity (0.4\% DDM, Fig. $5 \mathrm{~g}$ ). The stability of PSI tetramer, indicated by the relative quantity at higher detergent concentration (0.8\% DDM, Fig. $5 \mathrm{~g})$, also was enhanced by higher light intensities. In contrast, 
(a)

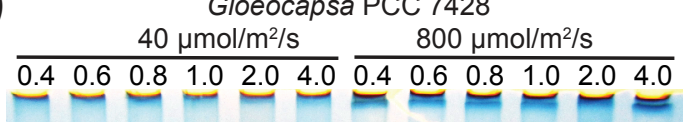

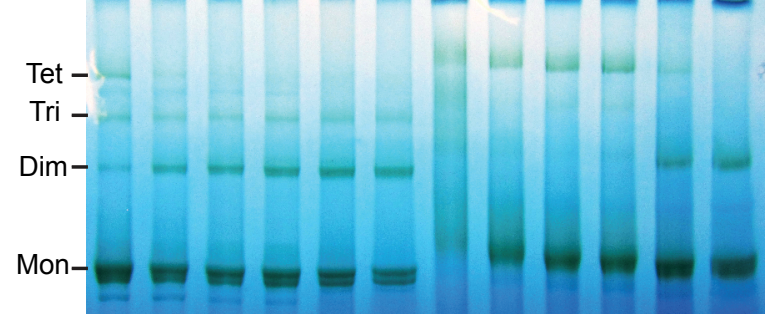

(b)

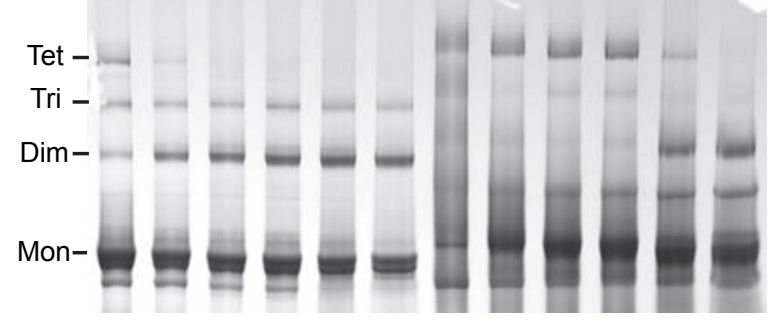

(c)

Fischerella PCC 7414
$\frac{40 \mu \mathrm{mol} / \mathrm{m}^{2} / \mathrm{s}}{0.40 .60 .81 .02 .04 .0} \frac{800 \mu \mathrm{mol} / \mathrm{m}^{2} / \mathrm{s}}{0.60 .81 .02 .04 .0}$

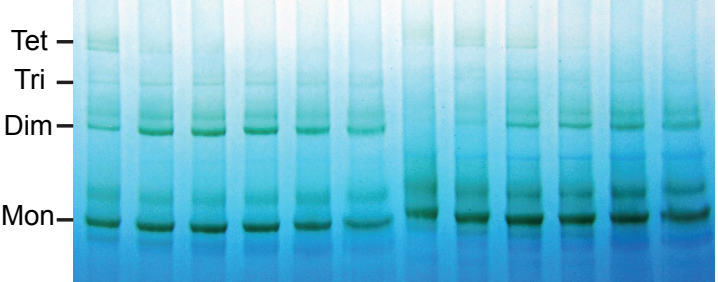

(d)

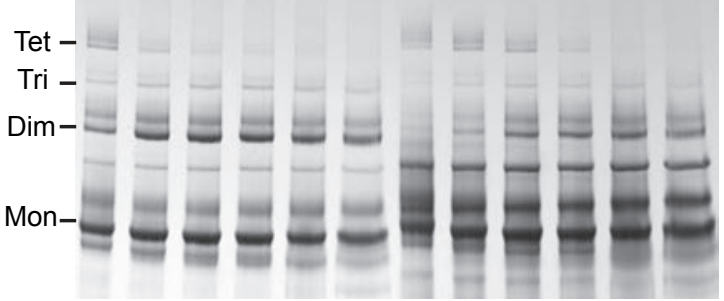

Fig. 4. BN-PAGE analyses of cyanobacterial PSI oligomeric states under different light

intensities. (a) and (c) show the unstained BN-PAGE analyses of solubilized thylakoid membranes from PCC 7428 and PCC 7414 respectively. The left lanes are the low light (40 $\left.\mu \mathrm{mol} / \mathrm{m}^{2} / \mathrm{s}\right)$ and the right lanes are the high light $\left(800 \mu \mathrm{mol} / \mathrm{m}^{2} / \mathrm{s}\right)$. (c) and (d) present the same BN-PAGE gels as a and $\mathbf{c}$ respectively after staining with Coomassie to enhance clarity. Thylakoid membranes with $0.2 \mathrm{mg} / \mathrm{mL}$ Chl were solubilized in different concentrations of DDM (w/v, \%), labeled on top of each lane. 1.6 $\mu \mathrm{g} \mathrm{Chl}$ were loaded for each condition. Main bands corresponding to PSI monomer (Mon), Dimer (Dim), Trimer (Tri), and Tetramer (Tet) are labeled. 
(a)

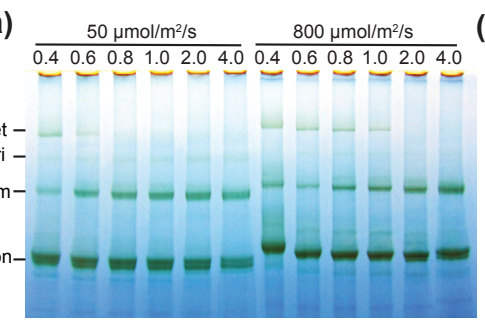

(c)

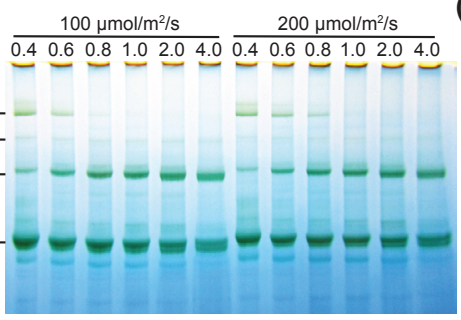

(e)
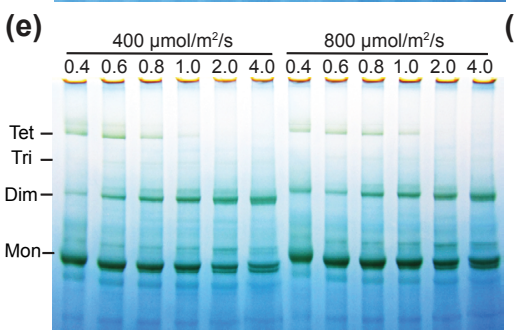

(g)

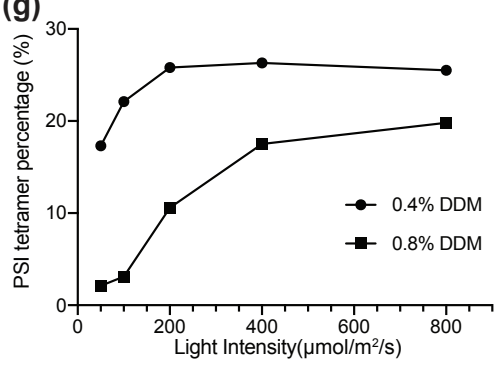

(b) $50 \mathrm{Hmol} / \mathrm{m}^{2} / \mathrm{s}$
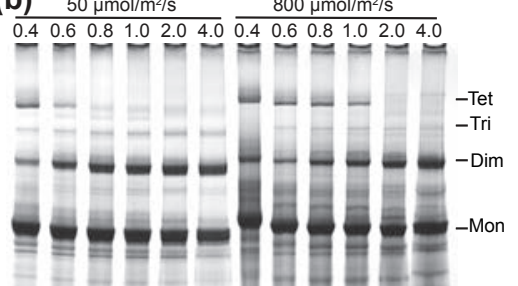

(d)

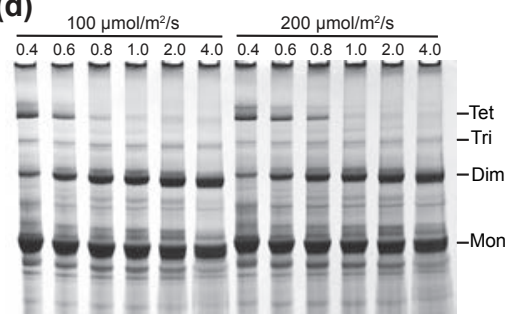

(f)

$\frac{400 \mu \mathrm{mol} / \mathrm{m}^{2} / \mathrm{s}}{0.40 .6 \quad 0.81 .02 .0 \quad 4.0} \frac{800 \mu \mathrm{mol} / \mathrm{m}^{2} / \mathrm{s}}{0.4 \quad 0.6 \quad 0.8 \quad 2.04 .0}$

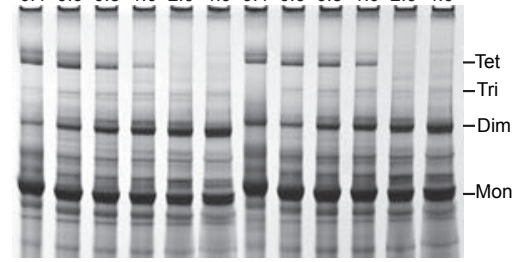

(h)

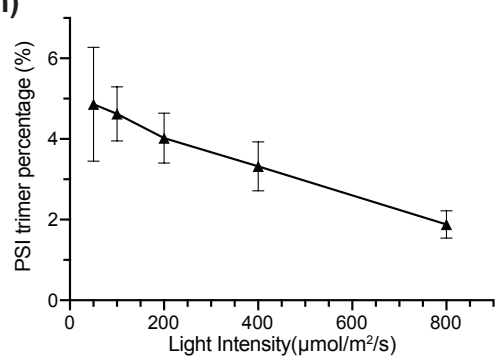

Fig. 5. Comparison of TS-821 PSI oligomeric profiles under different light intensities.

(a) (c) and (e) BN-PAGE of solubilized thylakoid membrane from TS-821 cultured under different light intensities. Coomassie stained images are shown as in (b) (d) and (f) for clarity and quantification. Thylakoid membranes with $0.2 \mathrm{mg} / \mathrm{mL} \mathrm{Chl} \mathrm{were} \mathrm{solubilized} \mathrm{in} \mathrm{different}$ concentrations of DDM (w/v, \%), labeled on top of each lane. $\sim 1.6 \mu \mathrm{g}$ Chl were loaded for each condition. Main bands corresponding to PSI monomer (Mon), dimer (Dim), trimer (Tri), and tetramer (Tet) are labeled. (g), Plots of relative quantities of PSI tetramer under different light intensities. Two solubilization conditions, $0.4 \%$ and $0.8 \%$ DDM, are presented to show the observed maximum quantities and the stabilities of PSI tetramer under different light intensities. (h), Plot of relative quantities of PSI trimer under different light intensities. Error bar: standard deviation ( $\mathrm{n}=5$ solubilization conditions). 
the relative amount of trimeric PSI decreases nearly linearly as the light intensity increases (Fig. 5h). This ability to alter the oligomeric form of PSI is not found in all species of Chroococcidiopsis, as indicated by the observation that the absence of PSI tetramer in PCC 7203 was not affected by light intensity (Fig. S7d). To investigate whether tetrameric PSI in TS-821 undergoes a shift in pigment composition or biochemical/biophysical changes under different light intensities, absorption spectra and $77 \mathrm{~K}$ fluorescence spectra were compared for different PSI oligomers isolated from cells grown under different light conditions (Figs 6a, b, S9). While the 77K emission spectra of these isolated PSI oligomers did not show significant differences (Fig. S9), it is clear that the PSI tetramer under HL has an additional absorption in the range around 440-560 nm, compared with tetrameric PSI under LL (Fig. 6a, b). The difference spectrum from these two samples showed that at least three discernable peaks $(450,490$, and $520 \mathrm{~nm}$ ) (Fig. 6b). The absorption spectrum of the LL PSI tetramer also showed a similar additional absorption around 400-500 $\mathrm{nm}$ when compared with the PSI trimer under LL (Fig. S9).

Interestingly, when the PSI tetramer is treated with 5\% DDM and dissociated into dimers, a very prominent amount of carotenoids stayed on the top layer of the sucrose gradient after separation by centrifugation (Fig. 6c). It is very likely that these carotenoids are associated with the interdimer interface and may even reside in the center of the tetrameric structure, only being released upon dissociation. Interestingly, the absorption spectrum of released carotenoids (Fig. 6d) resembled the increased absorbance observed in the HL tetramer (Fig. 6b). Moreover, spectral analysis of the tetrameric PSI in both TS-821 and PCC 7414 reveal an increase in absorption in the range of $400 \sim 550 \mathrm{~nm}$ when compared with the PSI dimer and trimer (Fig. S10c, d), and is quite similar to the inter-dimer carotenoids absorption spectrum (Fig. 6d). These results suggest that tetrameric forms of PSI are generally rich in carotenoids, compared to trimeric and dimeric forms of PSI. When these inter-dimer carotenoids were isolated and analyzed by HPLC, we identified novel PSI cofactors: myxoxanthophyll, canthaxanthin, echinenone, in addition to known cofactors: chlorophyll (Chl) and $\beta$-carotene (Figs. 6e, S11a). The semi-quantitative comparison between the HL PSI tetramer and LL PSI tetramer also revealed that tetrameric PSI 
(a)

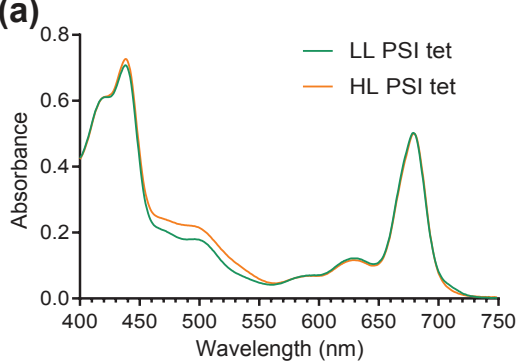

(c)

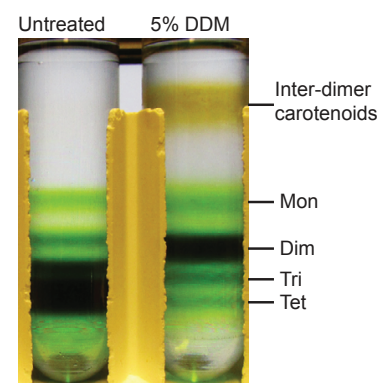

(e)

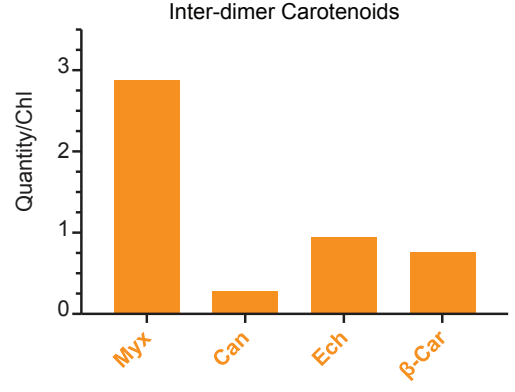

(b)

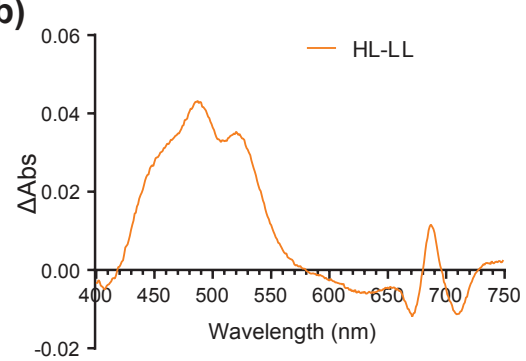

(d)

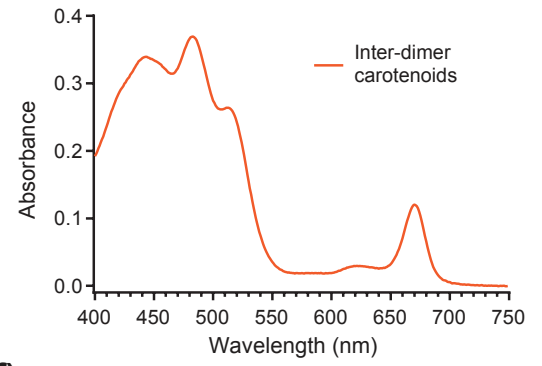

(f)

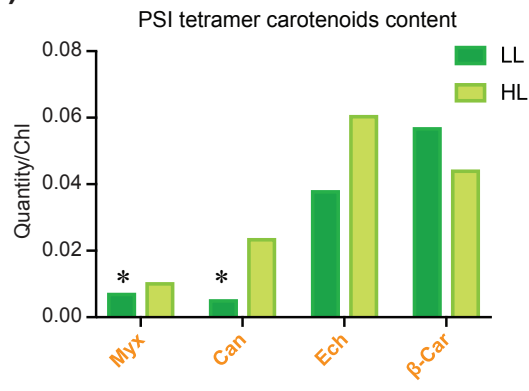

Fig. 6. The impact of light intensities on tetrameric PSI in TS-821. (a) Absorption spectra of PSI tetramer isolated from TS-821 grown under low light (LL) and high light (HL). (b) Absorption spectrum difference between HL and LL PSI tetramer calculated from spectra a. (c) Sucrose density gradient ultracentrifugation of isolated PSI tetramer. The sample treated with 5\% DDM is compared with untreated PSI tetramer. Main bands corresponding to inter-dimer carotenoids, PSI monomer (Mon), Dimer (Dim), Trimer (Tri), and Tetramer (Tet) are labeled. (d) Absorption spectrum of inter-dimer carotenoids isolated from treated PSI tetramer after sucrose density gradient ultracentrifugation c. (e) Quantitative analysis of carotenoids released upon inter-dimer carotenoids. (f) Quantitative comparison of carotenoids in PSI tetramer isolated under high light (HL) and low light (LL) conditions. The ratios of carotenoids to Chl are presented. The detected carotenoids include myxoxanthophyll (Myx), canthaxanthin (Can), echinenone (Ech) and $\beta$-carotene ( $\beta$-Car). Asterisks $(*)$ denote the detected quantities are below

346 limit of quantification. 


\section{Discussion}

349 Our results indicate that tetrameric PSI is a characteristic shared by most, if not all, heterocyst-

350 forming cyanobacteria and their closest unicellular relatives. The distribution of tetrameric PSI in

351 this clade of cyanobacteria points out a shared physiological characteristic between the two

352 morphologically distinct cyanobacteria. The prior classification of PCC 7428 and TS-821 would

353 place these cyanobacteria in Section II based on their reproduction by multiple fission giving rise

354 to baeocytes (Rippka et al., 1979), but our current work on these PSI-dimer/tetramer containing

355 unicellular cyanobacteria would support a different placement. Phylogenetic analyses based on

356 16S rRNA gene as well as a more universal set of conserved genes suggest that these tetramer-

357 forming cyanobacteria are closely related to heterocyst-forming cyanobacteria (Fewer et al.,

358 2002; Shih et al., 2013). However, no further physiological or biochemical evidence had been

359 reported to support the close evolutionary linkage between the two groups. In this study, we

360 showed similar physiological and biochemical properties of the PSI oligomers observed in those

361 two groups, providing further support of a phylogenetic placement of the PSI-dimer/tetramer

362 unicellular cyanobacteria close to heterocyst-forming strains, as one clade, HCR.

363 Additionally, the phylogenetic analysis provided a clear classification of PsaL in correlation to

364 PSI oligomeric state (Fig. S4). With given cyanobacterial psaL sequences, the major PSI

365 oligomeric states can be predicted, even though specific strains may vary from the major

366 correlation between PsaL and PSI oligomer, such as PSI monomer found in heterocyst-forming

367 cyanobacteria (Fig. S1). The formation of the tetrameric structure may be a result of changes in

368 the PsaL subunit, as proposed in earlier work (Li et al., 2014), and it genomic location in relation

369 to $P s a F / J$ which can be an element for expression regulation and PSI assembly control. Yet

370 changes in PsaL are insufficient for PSI tetramer formation, consistent with the observations of

371 PSI monomer in PCC 6803 expressing TS-821 PsaL. Besides differences in PsaL, other factors

372 such as carotenoids and PSI assembly chaperones, may contribute to the formation of tetrameric

373 PSI.

374 The discovery of a pool of PSI inter-dimer carotenoids also suggests the physiological

375 significance of tetrameric PSI over trimeric PSI, since these additional carotenoids may protect

376 PSI from excessive influx of photons under HL. This inference is further supported by the fact

377 that the carotenoid content, as well as the relative quantity and stability of PSI tetramer increased

378 under HL (Figs 5, 6). Even under LL, PSI tetramer has higher content of carotenoids compared 
with PSI trimer (Fig. S10). This is in line with the observations of antennae cooperativity between the three T. elongatus PSI monomers to yield an increased optical cross-section of the trimer (Iwuchukwu et al., 2010; Baker et al., 2014), while tetrameric and dimeric PSI did not show significant cooperativity among the monomers (Li et al., 2014). Altogether, tetrameric PSI by itself, compared to trimeric PSI, is a less efficient form of PSI in light harvesting but a better form of PSI in terms of dissipating excessive photonic energy under HL conditions, thereby avoiding photoinhibition. Thus, these cyanobacteria can alter the oligomeric state of PSI as light increases, while also increasing the content of the photoprotective carotenoids. It is not clear if it is the increase in the carotenoids that facilitate the formation of the tetramer or vice versa, yet it is attractive to speculate that the tetrameric structure provides a mechanism to accumulate carotenoids possibly via an unknown and/or novel carotenoid binding mechanism. The light responsive adaptive behavior of the PSI oligomeric state and the ability of a PSI tetramer adjusting its carotenoid content, represents an early evolutionary and unique photo-protective mechanism in HCR.

Our results support the hypothesis that PSI oligomers evolved from trimeric to tetrameric in cyanobacteria en route to exclusively monomeric in plants and green algae. By comparing PSI trimeric structure from cyanobacteria and PSI monomeric structure from plants, it was proposed that PSI evolved from a PSI trimer to PSI monomer due to the presence of PsaH, preventing the formation of PSI trimer (Amunts and Nelson, 2009). However, the presence of exclusively monomeric PSI in cyanobacteria (Fig. S2), red algae and heterokonts (Alboresi et al., 2017) without the involvement of PsaH suggests that PSI monomerization preceded the introduction of the PsaH subunit. Changes in PsaL appeared sufficient for PSI monomerization notably with Synechocystis expressing Arabidopsis PsaL exhibiting exclusively monomeric PSI (Fig. 3b). Therefore, the plant PsaL appeared more closely related to cyanobacterial PsaL in tetrameric/monomeric PSI. The presence of exclusively monomeric PSI in heterocyst-forming cyanobacteria (Fig. S2) or mostly monomeric PSI in examples such Chroococcidiopsis sp. PCC 7203 (Figs 1a, S2) suggests that the monomerization of PSI in plants and green algae shared the same or closely related route to that of PSI tetramer formation over the course of evolution. 
411 mainly trimeric PSI, accumulates more PSI monomer when grown under HL (Wang et al., 2008),

412 which suggests that monomeric PSI may also be advantageous over trimeric PSI under this

413 increased light intensity. Our study on Chroococcidiopsis sp. PCC 7203, which had mostly

414 monomeric PSI and some dimeric PSI, showed no obvious oligomeric shift when transferred

415 from LL to HL (Fig. S7). Whether monomeric PSI is a further adaptation to HL remains to be

416 investigated.

417 Nevertheless, it is reasonable to speculate that early plastid ancestors needed to be able to cope 418 with HL irradiance as they moved from relatively light limiting marine/aquatic settings onto the 419 surface of a terrestrial environment. Compared with the PSI trimer, the PSI tetramer or monomer 420 seem to be more adapted to such light intensity. These PSI oligomers are also well suited for the 421 addition of other PSI subunits such as PsaH and light harvesting complexes that bind to the inter422 monomer faces of PSI. Finally, the observation of a very similar PSI tetramer in the glaucophyte 423 Cyanophora paradoxa (Watanabe et al., 2011) suggests that the closest cyanobacterial relative to 424 plastid ancestors may have also had tetrameric PSI, reinforcing the gene homologies and 425 phylogenomic conclusions that heterocyst-forming cyanobacteria are the most closely related to 426 the plastid ancestor (Dagan et al., 2013). 


\section{Materials and Methods}

Cyanobacterial growth conditions

The regular growth conditions for three control strains, Synechocystis sp. PCC 6803 (WT and mutants), Chroococcidiopsis sp. TS-821 (TS-821) and T. elongatus BP-1 were described in earlier study (Li et al., 2014). Cyanobacteria from UTEX were maintained as recommended by

432 the culture collection center at University of Texas, Austin, USA, and the Pasteur Culture Collection of Cyanobacteria at the Institut Pasteur, Paris, France (Dataset S1). To achieve sufficient cell mass for PSI oligomer identification, the cyanobacteria were cultured in feasible media with light intensity in the range of $20 \sim 100 \mu \mathrm{mol}$ photons $/ \mathrm{m}^{2} / \mathrm{s}$ (Dataset S1). The modified ASN III medium contains BG-11 medium with addition of ASNIII media major salts (25 g/L $\mathrm{NaCl}, 3.5 \mathrm{~g} / \mathrm{L} \mathrm{MgSO}_{4} \times 7 \mathrm{H}_{2} \mathrm{O}, 2.0 \mathrm{~g} / \mathrm{L} \mathrm{MgCl}_{2}$ × $\left.6 \mathrm{H}_{2} \mathrm{O}, 0.5 \mathrm{~g} / \mathrm{L} \mathrm{KCl}\right)$.

To study the physiological significance of PSI tetramer in cyanobacteria, non-standard culture conditions were tested. To investigate the effect of nitrogen sources, some heterocystforming cyanobacteria were cultured separately in BG-11, BG-11 0 or BG-11+NH ${ }_{4}^{+}(\mathrm{BG}-11$ supplemented with $1 \mathrm{mM} \mathrm{NH}_{4} \mathrm{Cl}$ two days before cell harvesting). To test the effect of temperature, some thermophilic cyanobacteria were cultured at $45^{\circ} \mathrm{C}$ versus $37{ }^{\circ} \mathrm{C}$. In addition, a culture of Fischerella muscicola PCC 7414 grown at $37{ }^{\circ} \mathrm{C}$ was incubated at $24{ }^{\circ} \mathrm{C}$ before harvesting. To investigate the effect of salinity, some marine cyanobacteria were cultured in both BG-11 and modified ASNIII media.

The standard high light (HL) and low light (LL) comparison experiments for TS-821, PCC 7414, PCC 7428, and PCC 7203 were done as described before (Semchonok et al., 2016). To eliminate the potential stress from cell culture density and/or difference from lighting sources, a systematic analysis of how TS-821 respond to different level of light intensity was done by culturing similar inoculum in photobioreactor for two days under five light conditions $(50,100$, 200, 400 and $800 \mu \mathrm{mol}$ photons $/ \mathrm{m}^{2} / \mathrm{s}$ ). For each light condition, half of the light intensity was

452 provided by white LED light and the other half was from red LED. The mid-log phase TS-821 cell cultures $\left(\mathrm{OD}_{680}\right.$ 0.6-0.8) under white light fluorescent light were inoculated to the $25 \mathrm{~L}$

454 bioreactor to starting $\mathrm{OD}_{680}$ at 0.04 (measured by equipped fluorometer probe from Photon Systems Instruments, Drazov, Czech Republic) before turning on the bioreactor lights. Cells were harvested and kept in $-20^{\circ} \mathrm{C}$ before PSI oligomer analysis.

Thylakoid membrane isolation 
For thylakoid membrane preparation, cells are lysed by French Press. Cyanobacterial cell pellets were washed in buffer A (50 mM MES-NaOH, pH 6.5, $\left.5 \mathrm{mM} \mathrm{CaCl}_{2}, 10 \mathrm{mM} \mathrm{MgCl}_{2}\right)$ and then pelleted by centrifugation (Watanabe et al., 2009). Cell pellets were resuspended in lysis buffer (buffer A containing 0.5 M sorbitol) and homogenized before French Press. In the case of Scytonema crispum UTEX LB 1556, cell aggregates were broken into small fragments by grinding in liquid nitrogen before resuspension in lysis buffer and homogenization.

Homogenized cell suspensions were processed through the French Press three times at 1500 or $2000 \mathrm{psi}$ (10 to $14 \mathrm{MPa}$ ), then unbroken cells were removed by pelleting at 10,000 $\mathrm{g}$ for $5 \mathrm{~min}$.

In most cases, thylakoid membrane pelleting from cell lysate was done by centrifugation at 40,000 rpm (193,000 g Type 50.2 Ti, Beckman) for $30 \mathrm{~min}$ at $4{ }^{\circ} \mathrm{C}$. Thylakoid membrane pellets were resuspended in buffer $\mathrm{A}+12.5 \%$ glycerol and homogenized before storing at $-20{ }^{\circ} \mathrm{C}$ or -80 ${ }^{\circ} \mathrm{C}$. For HL versus LL experiments, thylakoids were washed in buffer A once and pelleted at $193,000 \mathrm{~g}$ for $15 \mathrm{~min}$ before final resuspension. In some special cases where cell mass is low and large membrane fragments are ready to be pelleted at lower g-force, thylakoids were resuspended after spinning at 10,000 $\mathrm{g}$ for $5 \mathrm{~min}$. Chl concentration was determined as described previously (Iwamura et al., 1970).

In most cases, 4-16\% BN-PAGE gels (Invitrogen) were used to analyze solubilized thylakoids or isolated photosystems according to the user manual and references (Schägger and von Jagow, 1991; Wittig et al., 2006). In addition, some of the BN-PAGE gels were homemade to analyze samples with large loading amount. To analyze PSI oligomeric states, thylakoid membranes were solubilized in different concentration of detergent $n$-dodecyl- $\beta$-maltoside (DDM) (Glycon, Germany) at $25^{\circ} \mathrm{C}$ for $1.5 \mathrm{~h}$. Insoluble material was removed by centrifuging $180,000 \mathrm{~g}$ for $5 \mathrm{~min}$ or $98,000 \mathrm{~g}$ for $10 \mathrm{~min}$ at $4{ }^{\circ} \mathrm{C}$. Supernatants were taken out for BN-PAGE analysis. To identify the photosystems after BN-PAGE, a second dimension Tris-Tricine SDSPAGE was used as described previously (Li et al., 2014).

To detect the presence of PsaL in Synechocystis mutants, Western blot was done after SDSPAGE. Protein bands were transferred from polyacrylamide gels to a $0.45 \mu \mathrm{m}$ PVDF membrane (Millipore) using blotting cassette (idEA, Minneapolis, MN). Membranes were blocked using TBS-T buffer $+3 \%$ NFM (non-fat milk powder) at room temperature for 1 hour. Primary antibody antisera were diluted from 1: 5000 in TBS-T +3\% NFM depending on the efficiency of antisera before treating the blocked membranes overnight at $4{ }^{\circ} \mathrm{C}$. Secondary antibody (goat anti- 
rabbit) conjugated to HRP was diluted in TBS-T + 3\% NFM down to 1:50,000 before treating the washed membranes for 1 hour at room temperature. The targeted proteins were then detected using chemiluminsence HRP substrate (Millipore) with signal recorded using ChemiDoc XDS system (Bio-Rad).

Antigen design and antibody production

PsaL sequences are not well conserved for antigen design from a single consensus sequence. To achieve one antigen for all PsaL of interest in this study, i.e. Chroococcidiopsis sp. TS-821, Arabidopsis, ․ . elongatus BP-1, and Synechocystis sp. PCC 6803, a fused protein, CATSPsaL containing fragments of different PsaL was designed. To predict the epitopes, Kolaskar \& Tongaonkar Antigenicity's methods (Kolaskar and Tongaonkar, 1990) on IEDB Analysis Resource (http://tools. immuneepitope.org/tools/bcell/iedb_input) and ABCpred (Saha and Raghava, 2006) (http://www.imtech. res.in/raghava/abcpred/index.html) were used. The antigen sequence is aligned against different PsaL fragments and its predicted antigen to ensure the high probability of getting antibody for each PsaL (Fig. S1). In addition to the N-terminal

504 fragments, the loop insertion between second and third trans-membrane helices of TS-821 PsaL was also used to achieve high antigenicity for TS-821.

Synthetic CATSpsaL gene (IDT, San Jose, CA) was cloned into pTYB2 (NEB) plasmid. The expression plasmid DNA was then transformed into E. coli ER2566 for antigen expression. IMPACT ${ }^{\mathrm{TM}}$ Chitin Resin (NEB) was used as column matrix for antigen purification. Antibody was produced at Pocono Rabbit Farm (Canadensis, PA) using the 91-day protocol on two rabbits.

510 For PsaL antibody production, the purified $1 \mathrm{mg} / \mathrm{mL}$ antigen was used as inoculum.

\section{$\underline{\text { PSI isolation and characterization }}$}

Sucrose Density Gradient Centrifugation (SDGC) was used for PSI isolation as described previously (Li et al., 2014). SDGC was also used to study PSI oligomeric states, as a parallel or complementary experiment to BN-PAGE profiling. Thylakoid membranes containing $0.4 \mathrm{mg} / \mathrm{mL}$ Chl were solubilized in 0.6 1\% DDM. After removing insoluble fragments, the solubilized

516 membranes containing $1 \mathrm{mg} \mathrm{Chl}$ were loaded on 10-30\% sucrose density gradient in buffer A containing 0.01\% DDM. This method showed comparable result with BN-PAGE analysis. For

518 large loading of solubilized membrane (containing 1 3 mg Chl) on a sucrose density gradient, centrifugation was done twice with the first 20 24-hour spin followed by dialysis and another

520 24-hour spin (SW32Ti, 30,000 rpm). Lower ionic strength such as $0.4 \mathrm{x}$ buffer A in sucrose density gradient for PCC 7414 PSI oligomers was tried and showed no observable difference for 
522 PSI profiles. Inter-dimer carotenoids were isolated after SDGC following treatment of PSI tetramer with 5\% DDM for at least one hour at room temperature. Isolated PSI samples were

524 dialyzed before analysis. For the proteomic comparison between PSI trimers and PSI tetramers, isolated PSI oligomers were further purified by BN-PAGE and sliced out for analyses.

Low temperature fluorescence spectra were acquired as described before (Li et al., 2014). Absorption spectra of isolated proteins and pigments were taken using Cary $300 \mathrm{UV}-\mathrm{V}$ is

528 spectrometer (Agilent). The sample buffers were used as blanks. For PSI oligomer absorption spectra comparisons, $\mathrm{A}_{680}$ was adjusted close to 0.5 or 1 . The PSI absorption differences were calculated after normalizing the data at $\mathrm{A}_{680}$. Electron microscopy and single particle analyses were done as described in previous studies (Li et al., 2014; Watanabe et al., 2014). Pigment analyses were done using HPLC by DHI (Denmark) (Van Heukelem L and Thomas CS, 2001). psaL gene cloning and TS-821 genome sequencing

Most cyanobacterial DNA extracted for $p s a L$ cloning were done as described in previous study (Li et al., 2014). TS-821 DNA for genome sequencing as well as some cyanobacterial DNA for $p s a L$ cloning was extracted using the NucleoBond ${ }^{\circledR}$ DNA isolation kit. For $p s a L$ cloning of heterocyst-forming cyanobacteria with unknown genome data, different primer sets

538 (Tables S1, S2) from $p s a F$ and gmk genes were used to amplify the $p s a L$ gene and its flanking region. The cyanobacteria of interests were classified using their known DNA sequences or genus names to facilitate picking primer pairs. PCR products were ligated into pJET vectors using CloneJET ${ }^{\text {тм }}$ PCR cloning kit. Plasmids containing cloned $p s a L$ were sequenced using

542 Sanger sequencing at UT Genomics Core (Knoxville, TN).

TS-821 culture was purified as described in earlier study (Rippka et al., 1979) before DNA 544 extraction for genome sequencing. The DNA was submitted to UT Genomic Core (Knoxville, TN) for sequencing using Illumina ${ }^{\circledR}$ MiSeq Kit V2 with 2 × 250 bp read length. The resulted

546 FASTQ files were analyzed using SPAdes (version 3.7.1) (Bankevich et al., 2012). The assembled contigs were submitted to PATRIC (Wattam et al., 2014) for annotation, which uses

548 the RAST (Overbeek et al., 2014) system.

Phylogenetic and psaL analyses

550 The species tree was generated by a concatenation of twenty-nine conserved proteins selected from the phylogenetic markers previously validated for cyanobacteria using a Maximum

552 Likelihood method as described previously (Calteau et al., 2014). Protein sequences were aligned using MAFFT v7.307 (Katoh and Standley, 2013), then ambiguous and saturated regions 
554 were removed with BMGE v1.12 (with the gap rate parameter set to 0.5) (Criscuolo and Gribaldo, 2010). The best fitting model of amino acid substitution for this dataset was selected

556 with ProtTest v3.2 (Darriba et al., 2011). A Maximum-Likelihood phylogenetic tree was generated with the alignment using PhyML 3.1.0.2 (Guindon et al., 2010) using the LG amino

558 acid substitution model with gamma-distributed rate variation (six categories), estimation of the proportion of invariable sites and exploring tree topologies. 100 bootstrap replicates were

560 performed. The phylogenetic trees were displayed and annotated using the interactive tree of life (iTOL) online tool (Letunic and Bork, 2016).

Genomic context of $\mathrm{psaL} / \mathrm{I} / \mathrm{F} / J$ genes and PsaL protein sequences were extracted from the MicroScope platform (Vallenet et al., 2017), public databases, and data generated in previous study (Schirrmeister et al., 2015) or this study (Dataset S1). The LOGO plot for the PsaL linker was generated using WebLogo 3 website (http://weblogo.threeplusone.com) (Crooks et al., 566 2004).

Construction of Synechocystis psaL mutants

568 Some psaL mutants were obtained in previous study (Li et al., 2014) with low expression efficiency. To improve the expression level of exogenous PsaL, the codon optimized synthetic psaL DNAs in pBSK vector were obtained from IDT (San Jose, CA). Similar methods were used for cloning and Synechocystis transformation as described earlier (Li et al., 2014). The mutants are named as Synechocystis sp. PCC 6803 expressing Synthetic Chroococcidiopsis sp. TS821/ㄹarabidopsis thaliana psaL (SCHL/SAtL). 


\section{Acknowledgments:}

576 Support to B.D.B., M.L., and J.T.N. has been provided from the Gibson Family Foundation, the Bredesen Center for Interdisciplinary Research and Education, the Tennessee Plant Research 578 Center, a UTK Professional Development Award, the Dr. Donald L. Akers Faculty Enrichment Fellowship to B.D.B. and National Science Foundation support to B.D.B. (DGE-0801470 and EPS-1004083). M.L. has been supported as a CIRE Fellow at University of Tennessee, Knoxville. A Professional Development Award from the Graduate School at UTK supported travel of B.D.B. to the Netherlands and to the Institut Pasteur. NWO Chemical Sciences supported work at University of Groningen. J.P.W. has been supported from NIH P30

584 DK063491. The Institut Pasteur supported Pasteur Culture Collection of Cyanobacteria. We are also grateful to Y.I. Park for the use of cyanobacterial genome of PCC 7124. We would like to thank Mr. Nathan G. Brady for helpful comments on the manuscript.

\section{Author contributions:}

M.L., M.G. and B.D.B designed the research. M.L. carried out most of the biochemistry and 590 molecular biology experiments. A.C did the phylogenetic and bioinformatics analyses. D.A.S and E.J.B did the EM imaging and single particle analyses. T.A.W did most of the psaL cloning.

592 N.S. and J.T.N. prepared most of the cell materials. J.T.N carried out the spectral comparison among PCC 7414 PSI oligomers J.W. did the proteomic analyses. M.L. and B.D.B wrote the article, while all other authors have contributed in editing and revising the article.

\section{Accession numbers:}

The cloned cyanobacterial psaL sequences can be found in GenBank through the following 598 accession numbers: KY575410, KY575411, KY575412, KY575413, KY575414, KY575415, KY575416, KY575417, KY575418, KY575419, KY575420, KY575421, KY575422,

600 KY575423, KY575424. TS-821 Whole Genome Shotgun project has been deposited at DDBJ/ENA/GenBank under the accession MVDI00000000. The version described in this paper 602 is version MVDI01000000. 


\section{References:}

608

614

Alboresi A, Le Quiniou C, Yadav SK, Scholz M, Meneghesso A, Gerotto C, Simionato D, Hippler M, Boekema EJ, Croce R et al. 2017. Conservation of core complex subunits shaped the structure and function of photosystem $I$ in the secondary endosymbiont alga Nannochloropsis gaditana. New Phytol. 213: 714-726.

Almog O, Shoham G, Michaeli D, and Nechushtai R. 1991. Monomeric and trimeric forms of photosystem I reaction center of Mastigocladus laminosus: crystallization and preliminary characterization. Proc. Natl. Acad. Sci. USA 88: 5312-5316.

Amunts A, and Nelson N. 2009. Plant photosystem I design in the light of evolution. Structure

Baker DR, Manocchi AK, Lamicq ML, Li M, Nguyen K, Sumner JJ, Bruce BD, and Lundgren CA. 2014. Comparative photoactivity and stability of isolated cyanobacterial monomeric and trimeric photosystem I. J Phys. Chem. B 118: 2703-2711.

Bankevich A, Nurk S, Antipov D, Gurevich AA, Dvorkin M, Kulikov AS, Lesin VM, Nikolenko SI, Pham S, Prjibelski AD et al. 2012. SPAdes: A new genome assembly algorithm and its applications to single-cell sequencing. J Comput. Biol. 19: 455-477.

Ben-Shem A, Frolow F, and Nelson N. 2003. Crystal structure of plant photosystem I. Nature 426: 630-635.

Bibby TS, Mary I, Nield J, Partensky F, and Barber J. 2003. Low-light-adapted Prochlorococcus species possess specific antennae for each photosystem. Nature 424: 1051-1054.

Boekema EJ, Dekker JP, Vanheel MG, Rogner M, Saenger W, Witt I, and Witt HT. 1987. Evidence for a trimeric organization of the photosystem I complex from the thermophilic cyanobacterium Synechococcus sp. Febs Lett 217: 283-286.

Boekema EJ, Hifney A, Yakushevska AE, Piotrowski M, Keegstra W, Berry S, Michel KP, Pistorius EK, and Kruip J. 2001. A giant chlorophyll-protein complex induced by iron defciency in cyanobacteria. Nature 412: 745-748.

\section{Calteau A, Fewer DP, Latifi A, Coursin T, Laurent T, Jokela J, Kerfeld CA, Sivonen K,} Piel J, and Gugger M. 2014. Phylum-wide comparative genomics unravel the diversity of secondary metabolism in Cyanobacteria. BMC Genomics 15: 977.

Cardona T, Battchikova N, Zhang P, Stensjo K, Aro EM, Lindblad P, and Magnuson A. 2009. Electron transfer protein complexes in the thylakoid membranes of heterocysts 
from the cyanobacterium Nostoc punctiforme. Biochimica et Biophysica Acta. Bioenergetics 1787: 252-263.

Casella S, Huang F, Mason D, Zhao GY, Johnson GN, Mullineaux CW and Liu LN. 2017.

Dissecting the native architecture and dynamics of cyanobacterial photosynthetic machinery. Molecular Plant 10: 1434-1448.

Criscuolo A, and Gribaldo S. 2010. BMGE (Block Mapping and Gathering with Entropy): A new software for selection of phylogenetic informative regions from multiple sequence alignments. BMC Evol Biol 10: 210.

Crooks GE, Hon G, Chandonia JM, and Brenner SE. 2004. WebLogo: A sequence logo generator. Genome Res 14: 1188-1190.

Dagan T, Roettger M, Stucken K, Landan G, Koch R, Major P, Gould SB, Goremykin VV, Rippka R, Tandeau de Marsac $\mathbf{N}$ et al. 2013. Genomes of Stigonematalean cyanobacteria (subsection $\mathrm{V}$ ) and the evolution of oxygenic photosynthesis from prokaryotes to plastids. Genome Biol. Evol. 5: 31-44.

Darriba D, Taboada GL, Doallo R, and Posada D. 2011. ProtTest 3: Fast selection of best-fit models of protein evolution. Bioinformatics 27: 1164-1165.

Fewer D, Friedl T, and Budel B. 2002. Chroococcidiopsis and heterocyst-differentiating cyanobacteria are each other's closest living relatives. Mol Phylogenet Evol 23: 82-90.

Gan F, and Bryant DA. 2015. Adaptive and acclimative responses of cyanobacteria to far-red light. Environ. Microbiol. 17: 3450-3465.

Gan F, Zhang S, Rockwell NC, Martin SS, Lagarias JC, and Bryant DA. 2014. Extensive remodeling of a cyanobacterial photosynthetic apparatus in far-red light. Science $\mathbf{3 4 5}$ : 1312-1317.

Garczarek L, van der Staay GWM, Thomas JC, and Partensky F. 1998. Isolation and characterization of Photosystem I from two strains of the marine oxychlorobacterium Prochlorococcus. Photosynth Res 56: 131-141.

Gardian Z, Bumba L, Schrofel A, Herbstova M, Nebesarova J, and Vacha F. 2007. Organisation of Photosystem I and Photosystem II in red alga Cyanidium caldarium: Encounter of cyanobacterial and higher plant concepts. Biochimica et Biophysica Acta Bioenergetics 1767: 725-731.

Golbeck JH. 2007. Photosystem I: The light-driven plastocyanin: ferredoxin oxidoreductase. Springer Netherlands. 
Guindon S, Dufayard JF, Lefort V, Anisimova M, Hordijk W, and Gascuel O. 2010. New

672

674

676

678

680

682

684

686

688

690

algorithms and methods to estimate maximum-likelihood phylogenies: Assessing the performance of PhyML 3.0. Syst Biol 59: 307-321.

Iwamura T, Nagai H, and Ichimura S-E. 1970. Improved methods for determining contents of chlorophyll protein bibonucleic acid and deoxyribonucleic acid in planktonic populations. Internationale Revue der gesamten Hydrobiologie und Hydrographie 55: 131-147.

Iwuchukwu IJ, Vaughn M, Myers N, O'Neill H, Frymier P, and Bruce BD. 2010. Selforganized photosynthetic nanoparticle for cell-free hydrogen production. Nat. Nanotechnol. 5: 73-79.

Jordan P, Fromme P, Witt HT, Klukas O, Saenger W, and Krauss N. 2001. Threedimensional structure of cyanobacterial photosystem I at 2.5 A resolution. Nature 411: 909-917.

Katoh K, and Standley DM. 2013. MAFFT multiple sequence alignment software version 7: Improvements in performance and usability. Mol Biol Evol 30: 772-780.

Kolaskar AS, and Tongaonkar PC. 1990. A semi-empirical method for prediction of antigenic determinants on protein antigens. Febs Lett 276: 172-174.

Kouril R, van Oosterwijk N, Yakushevska AE, and Boekema EJ. 2005. Photosystem I: a search for green plant trimers. Photochemical \& Photobiological Sciences 4: 1091-1094.

Letunic I, and Bork P. 2016. Interactive tree of life (iTOL) v3: An online tool for the display and annotation of phylogenetic and other trees. Nucleic Acids Res 44: W242-245.

Li M, Semchonok DA, Boekema EJ, and Bruce BD. 2014. Characterization and evolution of tetrameric photosystem I from the thermophilic cyanobacterium Chroococcidiopsis sp. TS-821. Plant Cell 26: 1230-1245.

MacGregor-Chatwin C, Sener M, Barnett SF, Hitchcock A, Barnhart-Dailey MC, Maghlaoui K, Barber J, Timlin JA, Schulten K and Hunter CN. 2017. Lateral segregation of photosystem I in cyanobacterial thylakoids. Plant Cell 29: 1119-1136.

Malavath T, Caspy I, Netzer-EI SY, Klaiman D, Nelson N. 2018. Structure and function of wild-type and subunit-depleted photosystem I in Synechocystis. Biochimica et Biophysica Acta - Bioenergetics 1859: 645-654.

Mangels D, Kruip J, Berry S, Rogner M, Boekema EJ, and Koenig F. 2002. Photosystem I from the unusual cyanobacterium Gloeobacter violaceus. Photosynth Res 72: 307-319. 
Mazor Y, Borovikova A, and Nelson N. 2015. The structure of plant photosystem I supercomplex at 2.8 A resolution. Elife 4: e07433. Parrello B, Shukla M et al. 2014. The SEED and the Rapid Annotation of microbial genomes using Subsystems Technology. RAST. Nucleic Acids Res. 42: D206-D214.

Qin X, Suga M, Kuang T, and Shen JR. 2015. Photosynthesis. Structural basis for energy transfer pathways in the plant PSI-LHCI supercomplex. Science 348: 989-995.

Rippka R, Deruelles J, Waterbury JB, Herdman M, and Stanier RY. 1979. Generic Assignments Strain Histories and Properties of Pure Cultures of Cyanobacteria. J Gen Microbiol 111: 1-61.

Saha S, and Raghava GPS. 2006. Prediction of continuous B-cell epitopes in an antigen using recurrent neural network. Proteins 65: 40-48.

Schägger H, and von Jagow G. 1991. Blue native electrophoresis for isolation of membrane protein complexes in enzymatically active form. Analytical Biochemistry 199: 223-231.

714 Schirrmeister BE, Gugger M, and Donoghue PC. 2015. Cyanobacteria and the Great Oxidation Event: Evidence from genes and fossils. Palaeontology 58: 769-785.

Semchonok DA, Li M, Bruce BD, Oostergetel GT, and Boekema EJ. 2016. Cryo-EM structure of a tetrameric cyanobacterial photosystem I complex reveals novel subunit interactions. Biochimica et Biophysica Acta - Bioenergetics 1857: 1619-1626.

Shelaev IV, Mamedov MD, Gostev FE, Aybush AV, Li M, Nguyen J, Bruce BD, Nadtochenko VA. 2018. Comparisons of Electron Transfer Reactions in a Cyanobacterial Tetrameric and Trimeric Photosystem I Complexes. Photochem

Shih PM, Wu D, Latifi A, Axen SD, Fewer DP, Talla E, Calteau A, Cai F, Tandeau de Marsac N, Rippka $R$ et al. 2013. Improving the coverage of the cyanobacterial phylum using diversity-driven genome sequencing. Proceedings of the National Academy of Sciences 110: 1053-1058.

Shubin VV, Bezsmertnaya IN, and Karapetyan NV. 1992. Isolation from Spirulina Membranes of 2 Photosystem I-Type Complexes One of Which Contains Chlorophyll Responsible for the 77-K Fluorescence Band at 760 nm. Febs Lett 309: 340-342. 
Shubin VV, Tsuprun VL, Bezsmertnaya IN, and Karapetyan NV. 1993. Trimeric forms of the photosystem I reaction center complex pre-exist in the membranes of the cyanobacterium Spirulina platensis. Febs Lett 334: 79-82.

Vintila S and El-Shehawy R. 2010. Ammonium ions inhibit nitrogen fixation but do not affect heterocyst frequency in the bloom-forming cyanobacterium Nodularia spumigena strain AV1. Microbiology 153: 3704-3712

Vintila S and El-Shehawy R. 2010. Variability in the response of the cyanobacterium Nodularia spumigena to nitrogen supplementation. J. Environ. Monit. 12: 1885-1890

Tsiotis G, Haase W, Engel A, and Michel H. 1995. Isolation and structural characterization of trimeric cyanobacterial photosystem I complex with the help of recombinant antibody fragments. Eur J Biochem 231: 823-830.

Tucker DL, and Sherman LA. 2000. Analysis of chlorophyll-protein complexes from the cyanobacterium Cyanothece sp. ATCC 51142 by non-denaturing gel electrophoresis. Biochimica et Biophysica Acta - Biomembranes 1468: 150-160. Rollin J, Rouy Z et al. 2017. MicroScope in 2017: An expanding and evolving integrated resource for community expertise of microbial genomes. Nucleic Acids Res $\mathbf{4 5}$ : D517-D528.

Veith T, and Buchel C. 2007. The monomeric photosystem I-complex of the diatom Phaeodactylum tricornutum binds specific fucoxanthin chlorophyll proteins (FCPs) as light-harvesting complexes. Biochimica et Biophysica Acta - Bioenergetics 1767: 14281435.

Wang Q, Jantaro S, Lu B, Majeed W, Bailey M, and He Q. 2008. The high light-inducible polypeptides stabilize trimeric photosystem I complex under high light conditions in Synechocystis PCC 6803. Plant Physiol. 147: 1239-1250.

Watanabe M, Iwai M, Narikawa R, and Ikeuchi M. 2009. Is the photosystem II complex a monomer or a dimer? Plant and Cell Physiology 50: 1674-1680.

Watanabe M, Kubota H, Wada H, Narikawa R, and Ikeuchi M. 2011. Novel supercomplex organization of photosystem I in Anabaena and Cyanophora paradoxa. Plant and Cell Physiology 52: 162-168. Ohmori M, Boekema EJ, and Ikeuchi M. 2014. Attachment of phycobilisomes in an 
antenna-photosystem I supercomplex of cyanobacteria. Proc. Natl. Acad. Sci. USA 111: 2512-2517.

764 Wattam AR, Abraham D, Dalay O, Disz TL, Driscoll T, Gabbard JL, Gillespie JJ, Gough R, Hix D, Kenyon R et al. 2014. PATRIC, the bacterial bioinformatics database and analysis resource. Nucleic Acids Res. 42: D581-591.

Wittig I, Braun H-P, and Schagger H. 2006. Blue native PAGE Nat. Protocols 1: 418-428. Hunter CN, and Johnson MP. 2018. Dynamic thylakoid stacking regulates the balance between linear and cyclic photosynthetic electron transfer. Nature Plants. 4: 116-127. https://doi.org/10.1038/s41477-017-0092-7

772 Van Heukelem L, and Thomas CS. 2001. Computer assisted high-performance liquid chromatography method development with applications to the isolation and analysis of phytoplankton pigments. J.Chromatogr. A. 910: 31-49. 


\section{Supporting Information}

778 Additional supporting information may be found in the online version of this article.

Fig. S1. PsaL antigen design and epitope prediction.

780 Fig. S2. Identification of PSI oligomers in heterocyst-forming cyanobacteria and their unicellular close relatives, contrasted by a few other cyanobacteria.

782 Fig. S3. PAGE analyses of PSI oligomers in cyanobacteria that are evolutionarily distant from heterocyst-forming cyanobacteria, contrasted by a few heterocyst-forming cyanobacteria

784 and close relatives.

Fig. S4. Species tree of cyanobacteria and Maximum-likelihood phylogenetic tree of PsaL.

786 Fig. S5. Screenshots of PsaL identification in PSI trimers and tetramers from PCC 7414, PCC 7428 and TS-821.

788 Fig. S6. LOGO plot and alignment of PsaL loop insertion between second and third transmembrane helices $(\mathrm{TMH})$.

790 Fig. S7. Effect of environmental factors on PSI oligomeric states in heterocystforming cyanobacteria and their close relatives.

792 Fig. S8. Macroscopic and microscopic images of strain PCC 7414.

Fig. S9. Spectral properties of PSI oligomers in TS-821 under different light

794 intensities.

Fig. S10. Absorption spectra of different PSI oligomers from TS-821 and PCC 7414.

796 Fig. S11. HPLC chromatograms of pigment analyses.

Table S1. PCR primers and conditions for different psaL cloning.

798 Table S2. Primer sequences used for psaL cloning.

Dataset S1. Summary of cyanobacterial PSI oligomeric states, culture conditions, and genomes studied. 